\title{
LAMB'S PLANE PROBLEM IN A THERMO-VISCO-ELASTIC MICROPOLAR MEDIUM WITH THE EFFECT OF GRAVITY
}

\author{
PRAVANGSU SEKHAR DAS
}

KTPP Township, Midnapore - 721171, India

\section{P.R. SENGUPTA}

Department of Mathematics University of Kalyani, Kalyani, West Bengal, India

and

\section{LOKENATH DEBNATH}

Department of Mathematics

University of Central Florida Orlando, Florida 32816, U.S.A.

(Received in September 1, 1990 and in revised form May 15, 1991)

\begin{abstract}
A study is made of the Lamb plane problem in an infinite thermo-visco-elastic micropolar medium with the effect of gravity. The visco-elasticity is characterized by the rate dependent theory of micro-visco-elasticity generalizing the classical Kelvin-Voigt theory. The action of time harmonic loading is treated in detail. The solutions for the displacement fields, couple stresses and the temperature field are obtained in general and particular cases.
\end{abstract}

Key Words and Phrases: Lamb's problem, Fourier transforms and effect of gravity. 1991 AMS Mathematics Subject Classification Code(s): 73D30

1. INTRODUCTION. Eringen [1-4] has developed a general theory of linear micropolar continuous media and of linear micropolar visco-elastic media. The classical Lamb problem in an elastic medium has received considerable attention in various elastic media with different kinds of loading. A selected reference including Sengupta and his associates [5-7], Chadha et al. [8], Rajneesh Kumar et al. [9] is cited for the reader.

The purpose of this paper is to study the effect of gravity on Lamb's plane problem in a micropolar thermo-visco-elastic medium. As far as we know, this problem has not yet received any attention.

2. FORMULATION OF THE PROBLEM. We consider a homogeneous micropolar thermo-viscoelastic semi-infinite medium with the influence of gravity under the action of loading $g\left(x_{1}, t\right)$ free plane boundary $x_{3}=0$. It is assumed that the medium is free to exchange heat with the material in the region $x_{3}>0$. It is everywhere at the constant absolute temperature $T_{0}$ prior to the appearance of any disturbance. 
Since we consider the plane problem, we assume that the displacement $\underline{u}=\left(u_{1}, 0, u_{3}\right)$ and rotation $\underline{\omega}=\left(0, \omega_{2}, 0\right)$ which are functions of $\left(x_{2}, x_{3}, t\right)$. The displacements are related to the displacement potentials $\phi\left(x_{1}, x_{3}, t\right)$ and $\psi\left(x_{1}, x_{3}, t\right)$ as follows:

$$
u_{1}=\phi_{x_{1}}-\psi_{x_{3}}, \quad u_{3}=\phi_{x_{3}}+\psi_{x_{1}}
$$

so that

$$
e=\nabla^{2} \phi, \quad \nabla^{2} \Psi=\frac{\partial u_{1}}{\partial x_{3}}-\frac{\partial u_{3}}{\partial x_{1}}
$$

where

$$
e=\frac{\partial u_{1}}{\partial x_{1}}+\frac{\partial u_{3}}{\partial x_{3}} \text { and } \nabla^{2}=\frac{\partial^{2}}{\partial x_{1}^{2}}+\frac{\partial^{2}}{\partial x_{3}^{2}}
$$

The basic dynamical equations of motion in a micropolar-visco-elastic solid medium under the influence of temperature are

$$
\begin{aligned}
& {\left[\left(\mu_{0}+\alpha_{0}\right)+\left(\mu_{1}+\alpha_{1}\right) \frac{\partial}{\partial t}\right] \nabla^{2} \underline{u}+\left[\left(\lambda_{0}+\mu_{0}-\alpha_{0}\right)+\left(\lambda_{1}+\mu_{1}-\alpha_{1}\right) \frac{\partial}{\partial t}\right] \operatorname{grad} d i v \underline{u}} \\
& +2\left(\alpha_{0}+\alpha_{1} \frac{\partial}{\partial t}\right) r o t \underline{\omega}-\left[\left(3 \lambda_{0}+2 \mu_{0}\right)+\left(3 \lambda_{1}+2 \mu_{1}\right) \frac{\partial}{\partial t}\right] \alpha_{1} \operatorname{grad} \theta=\rho \ddot{u} \\
& {\left[\left(\gamma_{0}+\varepsilon_{0}\right)+\left(\gamma_{1}+\varepsilon_{1}\right) \frac{\partial}{\partial t}\right] \nabla^{2} \underline{\omega}+\left[\left(\gamma_{0}+\beta_{0}-\varepsilon_{0}\right)+\left(\gamma_{1}+\beta_{1}-\varepsilon_{1}\right) \frac{\partial}{\partial t} \operatorname{grad} \operatorname{div} \underline{\omega}\right.} \\
& -4\left(\alpha_{0}+\alpha_{1} \frac{\partial}{\partial t}\right) \underline{\omega}+2\left(\alpha_{0}+\alpha_{1} \frac{\partial}{\partial t}\right) \operatorname{rot} \underline{u}=J \underline{\ddot{\omega}}
\end{aligned}
$$

where $\lambda_{0}, \mu_{0}$ are the Lame elastic constants while $\alpha_{0}, \beta_{0}, \gamma_{0}, \varepsilon_{0}$ are the other material constants, $\lambda_{1}, \mu_{1}, \alpha_{1}, \beta_{1}, \gamma_{1}, \varepsilon_{1}$ are the parameters representing the effects of viscosity. Also these elastic moduli $\lambda_{0}, \mu_{0}, \alpha_{0}, \beta_{0}, \gamma_{0}, \varepsilon_{0}$ and viscosities $\lambda_{1}, \mu_{1}, \alpha_{1}, \beta_{1}, \gamma_{1}, \varepsilon_{1}$ are subjected to the following restrictions

$$
\begin{aligned}
& 0 \leq 3 \lambda_{0}+2 \mu_{0}+\alpha_{0}, \quad 0 \leq 3 \beta_{0}+2 \gamma_{0}, \quad 0 \leq \mu_{0}, \quad-\gamma_{0} \leq \varepsilon_{0} \leq \gamma_{0}, \quad 0 \leq \alpha_{0}, \quad 0 \leq \gamma_{0} \\
& 0 \leq 3 \lambda_{1}+2 \mu_{1}+\alpha_{1}, \quad 0 \leq 3 \beta_{1}+2 \gamma_{1}, \quad 0 \leq \mu_{1}, \quad-\gamma_{1} \leq \varepsilon_{1} \leq \gamma_{1}, \quad 0 \leq \alpha_{1}, \quad 0 \leq \gamma_{1}
\end{aligned}
$$

and $\alpha_{\text {, }}$ is the coefficient of linear expansion of solid, $\rho$ is the density, $\theta=T-T_{0}(=$ absolute temperature-initial absolute temperature), and $J$ is the rotational inertia.

For the present study, it is convenient to rewrite equations (2.4)-(2.5) as

$$
\begin{gathered}
{\left[\left(\mu_{0}+\alpha_{0}\right)+\left(\mu_{1}+\alpha_{1}\right) \frac{\partial}{\partial t}\right] \nabla^{2} u_{1}+\left[\left(\lambda_{0}+\mu_{0}-\alpha_{0}\right)+\left(\lambda_{1}+\mu_{1}-\alpha_{1}\right) \frac{\partial}{\partial t}\right] \frac{\partial e}{\partial x_{1}}} \\
-2\left(\alpha_{0}+\alpha_{1} \frac{\partial}{\partial t}\right) \frac{\partial \omega_{2}}{\partial x_{3}}-\left[\left(3 \lambda_{0}+2 \mu_{0}\right)+\left(3 \lambda_{1}+2 \mu_{1}\right) \frac{\partial}{\partial t}\right] \alpha_{t} \frac{\partial \theta}{\partial x_{1}}+\rho g u_{3,1}=\rho \ddot{u}_{1} \\
{\left[\left(\mu_{0}+\alpha_{0}\right)+\left(\mu_{1}+\alpha_{1}\right) \frac{\partial}{\partial t}\right] \nabla^{2} u_{3}+\left[\left(\lambda_{0}+\mu_{0}-\alpha_{0}\right)+\left(\lambda_{1}+\mu_{1}-\alpha_{1}\right) \frac{\partial}{\partial t}\right] \frac{\partial e}{\partial x_{3}}} \\
+2\left(\alpha_{0}+\alpha_{1} \frac{\partial}{\partial t}\right) \frac{\partial \omega_{2}}{\partial x_{1}}-\left[\left(3 \lambda_{0}+2 \mu_{0}\right)+\left(3 \lambda_{1}+2 \mu_{1}\right) \frac{\partial}{\partial t}\right] \alpha_{t} \frac{\partial \theta}{\partial x_{3}}-\rho g u_{1,1}=\rho \ddot{u}_{3} \\
{\left[\left(\gamma_{0}+\varepsilon_{0}\right)+\left(\gamma_{1}+\varepsilon_{1}\right) \frac{\partial}{\partial t}\right] \nabla^{2} \omega_{2}+2\left(\alpha_{0}+\alpha_{1} \frac{\partial}{\partial t}\right)\left(\frac{\partial u_{1}}{\partial x_{3}}-\frac{\partial u_{3}}{\partial x_{1}}\right)-4\left(\alpha_{0}+\alpha_{1} \frac{\partial}{\partial t}\right) \omega_{2}-J \ddot{\omega}_{2}}
\end{gathered}
$$


The temperature $\theta$ satisfies the Fourier's law of heat conduction

$$
\kappa \nabla^{2} \theta=\rho C_{\varepsilon} \frac{\partial \theta}{\partial t}+T_{0}\left[\left(3 \lambda_{0}+2 \mu_{0}\right)+\left(3 \lambda_{1}+2 \mu_{1}\right) \frac{\partial}{\partial t}\right] \alpha_{t} \frac{\partial}{\partial t}\left(\nabla^{2} \phi\right),
$$

where $K$ is the thermal conductivity and $C_{\varepsilon}$ is the specific heat at constant strain.

Substituting (2.2ab) into (2.7)-(2.9) gives

$$
\begin{gathered}
{\left[\left(C_{1}^{2}+C^{\prime 2} \frac{\partial}{\partial t}\right) \nabla^{2}-\frac{\partial^{2}}{\partial t^{2}}\right] \phi+g \frac{\partial \psi}{\partial x_{1}}-\left(p_{1}^{2}+p_{1}{ }^{\prime 2} \frac{\partial}{\partial t}\right) \theta=0} \\
{\left[\left(C_{2}^{2}+C_{2}^{\prime 2} \frac{\partial}{\partial t}\right) \nabla^{2}-\frac{\partial^{2}}{\partial t^{2}}\right] \psi-g \frac{\partial \phi}{\partial x_{1}}+\left(p_{2}^{2}+p_{2}{ }^{\prime 2} \frac{\partial}{\partial t}\right) \omega_{2}=0} \\
{\left[\left(C_{4}^{2}+C_{4}{ }^{\prime 2} \frac{\partial}{\partial t}\right) \nabla^{2}-\left(C_{5}^{2}+C_{5}{ }^{2}+\frac{\partial^{2}}{\partial t^{2}}\right)\right] \omega_{2}-\frac{1}{2}\left(C_{5}^{2}+C_{5}{ }^{2} \frac{\partial}{\partial t}\right) \nabla^{2} \psi=0} \\
{\left[\left(C_{3}^{2} \nabla^{2}-\frac{\partial}{\partial t}\right)\right] \theta-r\left(p_{1}^{2}+p_{1}^{\prime 2} \frac{\partial}{\partial t}\right) \frac{\partial}{\partial t}\left(\nabla^{2} \phi\right)=0}
\end{gathered}
$$

where

$$
\begin{gathered}
C_{1}^{2}=\frac{\lambda_{0}+2 \mu_{0}}{\rho}, \quad C_{1}{ }^{\prime 2}=\frac{\lambda_{1}+2 \mu_{1}}{\rho}, \quad C_{2}^{2}=\frac{\mu_{0}+\alpha_{0}}{\rho}, \quad C_{2}{ }^{\prime 2}=\frac{\mu_{1}+\alpha_{1}}{\rho} \\
C_{3}^{2}=\frac{\kappa}{\rho C_{\varepsilon}}, \quad C_{4}^{2}=\frac{\gamma_{0}+\varepsilon_{0}}{J}, \quad C_{4}{ }^{\prime 2}=\frac{\gamma_{1}+\varepsilon_{1}}{J}, \quad C_{5}^{2}=\frac{4 \alpha_{0}}{J}, \quad C_{5}{ }^{2}=\frac{4 \alpha_{1}}{J} \\
p_{1}^{2}=\frac{\left(3 \lambda_{0}+2 \mu_{0}\right) \alpha_{t}}{\rho}, \quad p_{1}^{\prime 2}=\frac{\left(3 \lambda_{1}+2 \mu_{1}\right) \alpha_{t}}{\rho}, \quad p_{2}^{2}=\frac{2 \alpha_{0}}{\rho}, \quad p_{2}{ }^{\prime 2}=\frac{2 \alpha_{1}}{\rho}, \quad r=\frac{T_{0}}{C_{\varepsilon}}
\end{gathered}
$$

\section{BOUNDARY CONDITIONS.}

The stress-strain relations in considered medium are

$$
\begin{gathered}
\sigma_{i j}=\left[\left(\mu_{0}+\alpha_{0}\right)+\left(\mu_{1}+\alpha_{1}\right) \frac{\partial}{\partial t} \gamma_{i j}+\left[\left(\mu_{0}-\alpha_{0}\right)+\left(\mu_{1}-\alpha_{1}\right) \frac{\partial}{\partial t}\right] \gamma_{i j}\right. \\
+\left[\left(\lambda_{0}+\lambda_{1} \frac{\partial}{\partial t}\right) \gamma_{\kappa \kappa}-\left\{\left(3 \lambda_{0}+2 \mu_{0}\right)+\left(3 \lambda_{1}+2 \mu_{1}\right) \frac{\partial}{\partial t}\right\} \alpha_{t} \theta\right] \delta_{i j} \\
\mu_{i j}=\left[\left(\gamma_{0}+\varepsilon_{0}\right)+\left(\gamma_{1}+\varepsilon_{1}\right) \frac{\partial}{\partial t}\right] \chi_{i j}+\left[\left(\gamma_{0}+\varepsilon_{0}\right)+\left(\gamma_{1}-\varepsilon_{1}\right) \frac{\partial}{\partial t}\right] \chi_{i j}+\left(\beta_{0,}+\beta_{1} \frac{\partial}{\partial t}\right) \chi_{\kappa \mathrm{k}} \delta_{i j},
\end{gathered}
$$

in which

$$
\gamma_{j i}=u_{i, j}-\varepsilon_{\kappa j i} \omega_{\kappa}, \quad \chi_{j i}=\omega_{i, j} ;(i, j, \kappa=1,2,3)
$$

$\varepsilon_{\kappa j i}$ is the unit antisymmetric tensor, and $\sigma_{i j}$ is the Kronecker delta.

Hence the boundary conditions are

$$
\sigma_{33}=-f\left(x_{1}, t\right), \quad \sigma_{31}=0, \quad \mu_{32}=0 \quad \text { on } \quad x_{3}=0
$$

where

$$
\begin{aligned}
\sigma_{33}=2\left(\mu_{0}+\mu_{1} \frac{\partial}{\partial t}\right)\left[\phi_{33}-\psi_{13}\right] & +\left(\lambda_{0}+\lambda_{1} \frac{\partial}{\partial t}\right) \nabla^{2} \phi \\
& \left.-\left(3 \lambda_{0}+2 \mu_{0}\right)+\left(3 \lambda_{1}+2 \mu_{1}\right) \frac{\partial}{\partial t}\right] \alpha_{t} \theta
\end{aligned}
$$




$$
\begin{gathered}
\sigma_{31}=\left(\mu_{0}+\mu_{1} \frac{\partial}{\partial t}\right)\left[2 \phi_{13}+\psi_{33}-\psi_{11}\right]+\left(\alpha_{0}+\alpha_{1} \frac{\partial}{\partial t}\right)\left(\nabla^{2} \psi-2 \omega_{2}\right) \\
\mu_{32}=\left[\left(\gamma_{0}+\varepsilon_{0}\right)+\left(\gamma_{1}+\varepsilon_{1}\right) \frac{\partial}{\partial t}\right] \frac{\partial \omega_{2}}{\partial x_{3}}
\end{gathered}
$$

The thermal condition is

$$
\frac{\partial \theta}{\partial x_{3}}+h \theta=0 \text { on } x_{3}=0
$$

4. SOLUTION OF THE PROBLEM. We introduce Fourier's double integral transform pair defined by (see Debnath and Myint-U [10])

$$
\begin{aligned}
& \hat{\phi}\left(x_{3}, \xi, \eta\right)=\frac{1}{2 \pi} \int_{-\infty}^{\infty} \int\left(x_{1}, x_{3}, t\right) e^{i\left(x_{1} \xi+t \eta\right)} d x_{1} d t \\
& \phi\left(x_{1}, x_{3}, t\right)=\frac{1}{2 \pi} \int_{-\infty}^{\infty} \int \hat{\phi}\left(x_{3}, \xi, \eta\right) e^{-i\left(x_{1} \xi+(\eta)\right.} d \xi d \eta
\end{aligned}
$$

and similarly for $\hat{\psi}, \hat{\omega}_{2}, \hat{\theta}$.

Application of this transform reduces (2.10)-(2.13) into the form

$$
\begin{gathered}
{\left[\left(C_{1}^{2}-i \eta C_{1}^{\prime 2}\right)\left(\frac{d^{2}}{d x_{3}^{2}}-\xi^{2}\right)+\eta^{2}\right] \hat{\phi}-i \xi g \hat{\psi}-\left(p_{1}^{2}-i \eta p_{1}^{\prime 2}\right) \hat{\theta}=0} \\
{\left[\left(C_{2}^{2}-i \eta C_{2}^{\prime 2}\right)\left(\frac{d^{2}}{d x_{3}^{2}}-\xi^{2}\right)+\eta^{2}\right] \hat{\psi}+i \xi g \hat{\phi}-\left(p_{2}^{2}-i \eta p_{2}^{\prime 2}\right) \hat{\omega}_{2}=0} \\
{\left[\left(C_{4}^{2}-i \eta C_{4}^{\prime 2}\right)\left(\frac{d^{2}}{d x_{3}^{2}}-\xi^{2}\right)-\left(C_{5}^{2}-i \eta C_{5}^{\prime 2}-\eta^{2}\right)\right] \hat{\omega}_{2}-\frac{1}{2}\left(C_{5}^{2}-i \eta C_{5}^{\prime 2}\right)\left(\frac{d^{2}}{d x_{3}^{2}}-\xi^{2}\right) \hat{\psi}=0} \\
{\left[C_{3}^{2}\left(\frac{d^{2}}{d x_{3}^{2}}-\xi^{2}\right)+i \eta\right] \hat{\theta}+\operatorname{ri\eta }\left(p_{1}^{2}-i \eta p_{1}^{\prime 2}\right)\left(\frac{d^{2}}{d x_{3}^{2}}-\xi^{2}\right) \hat{\phi}=0}
\end{gathered}
$$

provided that in the case of exponential solutions of the equation the following conditions are satisfied

$$
\phi, \psi, \omega_{2}, \theta \rightarrow 0, \text { as } x_{3} \rightarrow \infty
$$

Therefore the solutions of the equations (4.2)-(4.5) given by

$$
\begin{aligned}
& \hat{\phi}=A e^{-x_{3} \sqrt{5_{1}}}+B e^{-x_{3} \sqrt{5_{2}}}+C e^{-x_{3} \sqrt{5_{5}}}+D e^{-x_{3} \sqrt{5_{4}}} \\
& \hat{\psi}=A_{1} e^{-x_{3} \sqrt{5_{1}}}+B_{1} e^{-x_{3} \sqrt{5_{2}}}+C_{1} e^{-x_{3} \sqrt{5_{5}}}+D_{1} e^{-x_{3} \sqrt{5_{1}}} \\
& \hat{\omega_{2}}=A_{2} e^{-x_{3} \sqrt{5_{1}}}+B_{2} e^{-x_{3} \sqrt{5_{2}}}+C_{2} e^{-x_{3} \sqrt{5_{5}}}+D_{2} e^{-x_{3} \sqrt{5_{4}}} \\
& \hat{\theta}=A_{3} e^{-x_{3} \sqrt{5_{1}}}+B_{3} e^{-x_{3} \sqrt{5_{2}}}+C_{3} e^{-x_{3} \sqrt{5_{3}}}+D_{3} e^{-x_{3} \sqrt{5_{4}}}
\end{aligned}
$$

where $\zeta_{j}^{2}(j=1,2,3,4)$, are the roots of the equation 


$$
\begin{aligned}
& \left\{\left(C_{1}^{2}-i \eta C_{1}{ }^{2}\right)\left(\zeta^{2}-\xi^{2}\right)+\eta^{2}\right\}\left[\{ ( C _ { 2 } ^ { 2 } - i \eta C _ { 2 } { } ^ { 2 } ) ( \zeta ^ { 2 } - \xi ^ { 2 } ) + \eta ^ { 2 } \} \left\{\left(C_{4}^{2}-i \eta C_{4}{ }^{2}\right)\left(\zeta^{2}-\xi^{2}\right)\right.\right. \\
& \left.\left.-\left(C_{5}^{2}-i \eta C_{5}{ }^{\prime 2}-\eta^{2}\right)\right\}\left\{C_{3}^{2}\left(\zeta^{2}-\xi^{2}\right)+i \eta\right\}-\frac{1}{2}\left(p_{2}^{2}-i \eta p_{2}{ }^{2}\right)\left(C_{5}^{2}-i \eta C_{5}{ }^{2}\right)\left(\zeta^{2}-\xi^{2}\right)\left\{C_{3}{ }^{2}\left(\zeta^{2}-\xi^{2}\right)+i \eta\right\}\right] \\
& -g^{2} \xi^{2}\left\{\left(C_{4}^{2}-i \eta C_{4}^{\prime 2}\right)\left(\zeta^{2}-\xi^{2}\right)-\left(C_{5}^{2}-i \eta C_{5}{ }^{2}-\eta^{2}\right)\right\}\left\{C_{3}^{2}\left(\zeta^{2}-\xi^{2}\right)+i \eta\right\} \\
& +\left(p_{1}^{2}-i \eta p_{1}^{\prime 2}\right)\left[\left\{\left(C_{2}^{2}-i \eta C_{2}^{\prime 2}\right)\left(\zeta^{2}-\xi^{2}\right)+\eta^{2}\right\}\right. \\
& \times\left\{\left(C_{4}^{2}-i \eta C_{4}{ }^{2}\right)\left(\zeta^{2}-\xi^{2}\right)-\left(C_{5}^{2}-i \eta C_{5}{ }^{2}-\eta^{2}\right)\right\}\left\{r i \eta\left(p_{1}^{2}-i \eta p_{1}{ }^{2}\right)\left(\zeta^{2}-\xi^{2}\right)\right\} \\
& \left.+\frac{1}{2} \operatorname{ri\eta }\left(C_{s}^{2}-i \eta C_{s}{ }^{2}\right)\left(p_{1}^{2}-i \eta p_{1}{ }^{2}\right)\left(\zeta^{2}-\xi^{2}\right)^{2}\right]=0
\end{aligned}
$$

and the constants $A, B, C, D, A_{1}, B_{1}, C_{1}, D_{1}, \ldots .$. etc. are related by

$$
\begin{array}{lll}
A_{1}=\alpha_{1}^{*} A, & A_{2}=\beta_{1}^{*} A, & A_{3}=\gamma_{1}^{*} A \\
B_{1}=\alpha_{2}^{*} B, & B_{2}=\beta_{2}^{*} B, & B_{3}=\gamma_{2}^{*} B \\
C_{1}=\alpha_{3}^{*} C, & C_{2}=\beta_{3}^{*} C, & C_{3}=\gamma_{3}^{*} C \\
D_{1}=\alpha_{4}^{*} D, & D_{2}=\beta_{4}^{*} D, & D_{3}=\gamma_{4}^{*} D
\end{array}
$$

where

$$
\begin{gathered}
\alpha_{j}=\frac{1}{i \xi g}\left[\left(C_{1}^{2}-i \eta C_{1}{ }^{2}\right)\left(\zeta_{j}-\xi^{2}\right)+\eta^{2}-\left(p_{1}^{2}-i \eta p_{1}{ }^{2}\right) \gamma_{j}^{*}\right] \\
\beta_{j}=\frac{\frac{1}{2}\left(C_{5}^{2}-i \eta C_{5}{ }^{2}\right)\left(\zeta_{j}-\xi^{2}\right) \alpha_{j}^{*}}{\left(C_{4}^{2}-i \eta C_{4}{ }^{\prime 2}\right)\left(\zeta_{j}-\xi^{2}\right)-\left(C_{5}^{2}-i \eta C_{5}{ }^{2}-\eta^{2}\right)} \\
\gamma_{j}=\frac{r i \eta\left(p_{1}^{2}-i \eta{p_{1}}^{2}\right)\left(\xi^{2}-\zeta_{j}\right)}{C_{3}^{2}\left(\zeta_{j}-\xi^{2}\right)+i \eta}, \quad(j=1,2,3,4)
\end{gathered}
$$

Substitution of (4.6)-(4.9), (4.1ab) into (3.4) yields

$$
\begin{aligned}
p_{1} A+p_{2} B+p_{3} C+p_{4} D & =-\hat{f}(\xi, \eta) \\
q_{1} A+q_{2} B+q_{3} C+q_{4} D & =0 \\
r_{1} A+r_{2} B+r_{3} C+r_{4} D & =0 \\
s_{1} A+s_{2} B+s_{3} C+s_{4} D & =0
\end{aligned}
$$

where

$$
\begin{gathered}
p_{j}=2\left(\mu_{0}-i \eta \mu_{1}\right)\left(\zeta_{j}-i \xi \alpha_{j}^{*} \sqrt{\zeta_{j}}\right)+\left(\lambda_{0}-i \eta \lambda_{1}\right)\left(\zeta_{j}-\xi^{2}\right) \\
-\left\{\left(3 \lambda_{0}+2 \mu_{0}\right)-i \eta\left(3 \lambda_{1}+2 \mu_{1}\right)\right\} \alpha_{1} \gamma_{j}^{*}, \\
q_{j}=\left(\mu_{0}-i \eta \mu_{1}\right)\left[\left(\zeta_{j}+\xi^{2}\right) \alpha_{j}^{*}+2 i \xi \sqrt{\zeta_{j}}+\left(\alpha_{0}-i \eta \alpha_{1}\right)\left[\alpha_{j}^{*}\left(\zeta_{j}-\xi^{2}\right)-2 \beta_{j}^{*}\right],\right. \\
\gamma_{j}=\beta_{j}^{*} \sqrt{\zeta_{j}}, \quad s_{j}=\gamma_{j}^{*}\left(\sqrt{\zeta_{j}}-h\right), \quad(j=1,2,3,4)
\end{gathered}
$$

Solving the equations (4.15abcd), we obtain

$$
A=\frac{\Delta_{1}}{\Delta} \hat{f}, \quad B=\frac{\Delta_{2}}{\Delta} \hat{f}, \quad C_{3}=\frac{\Delta_{3}}{\Delta} \hat{f}, \quad D=\frac{\Delta_{4}}{\Delta} \hat{f}
$$

where 


$$
\Delta=\left|\begin{array}{llll}
p_{1} & p_{2} & p_{3} & p_{4} \\
q_{1} & q_{2} & q_{3} & q_{4} \\
r_{1} & r_{2} & r_{3} & r_{4} \\
s_{1} & s_{2} & s_{3} & s_{4}
\end{array}\right|, \quad \Delta_{1}=\left|\begin{array}{cccc}
-1 & p_{2} & p_{3} & p_{4} \\
0 & q_{2} & q_{3} & q_{4} \\
0 & r_{2} & r_{3} & r_{4} \\
0 & s_{2} & s_{3} & s_{4}
\end{array}\right|
$$

Similarly, $\Delta_{2}, \Delta_{3}, \Delta_{4}$ are obtained, eliminating second, third and fourth column of $\Delta$ by the column $(-1,0,0,0)$.

Inserting the values of $A, B, C, D$ and (4.6)-(4.9) and using (4.1b) we obtain

$$
\begin{aligned}
& \phi=\frac{1}{2 \pi} \int_{-\infty}^{\infty} \int \frac{\hat{f}}{\Delta}\left[\Delta_{1} e^{-x_{3} \sqrt{\epsilon_{1}}}+\Delta_{2} e^{-x_{3} \sqrt{\xi_{2}}}+\Delta_{3} e^{-x \sqrt{\xi_{3}}}+\Delta_{4} e^{-x_{3} \sqrt{\varepsilon_{4}}}\right] e^{-i\left(x_{1} \xi+(\eta)\right.} d \xi d \eta(4.21) \\
& \psi=\frac{1}{2 \pi} \int_{-\infty}^{\infty} \int_{\Delta} \frac{\hat{f}}{\Delta}\left[\alpha_{1} \Delta_{1} e^{-x_{3} \sqrt{t_{1}}}+\alpha_{2}^{\infty} \Delta_{2} e^{-x_{3} \sqrt{\zeta_{2}}}+\alpha_{3}^{\infty} \Delta_{3} e^{-x_{3} \sqrt{\zeta_{3}}}+\alpha_{4}^{*} \Delta_{4} e^{-x_{3} \sqrt{\xi_{4}}}\right] e^{-i\left(x_{1} \xi+(\eta)\right.} d \xi d \eta \\
& \omega_{2}=\frac{1}{2 \pi} \int_{-\infty}^{\infty} \int_{\Delta}^{\infty} \frac{\hat{f}}{\Delta}\left[\beta_{1}^{*} \Delta_{1} e^{-x_{3} \sqrt{\xi_{1}}}+\beta_{2}^{*} \Delta_{2} e^{-x_{3} \sqrt{\varepsilon_{2}}}+\beta_{3}^{*} \Delta_{3} e^{-x_{3} \sqrt{\epsilon_{3}}}+\beta_{4}^{*} \Delta_{4} e^{-x_{3} \sqrt{\varepsilon_{1}}}\right] e^{-i\left(x_{1} \xi+(\eta)\right.} d \xi d \eta \\
& \theta=\frac{1}{2 \pi} \int_{-\infty}^{\infty} \int_{\Delta} \frac{\hat{f}}{\Delta}\left[\gamma_{1}^{*} \Delta_{1} e^{-x_{3} \sqrt{\varepsilon_{1}}}+\gamma_{2}^{*} \Delta_{2} e^{-x_{3} \sqrt{\varepsilon_{2}}}+\gamma_{3}^{*} \Delta_{3} e^{-x_{3} \sqrt{\xi_{3}}}+\dot{\gamma}_{4}^{*} \Delta_{4} e^{-x_{3} \sqrt{\varepsilon_{1}}}\right] e^{-i\left(x_{1} \xi+(\eta)\right.} d \xi d \eta
\end{aligned}
$$

We can now easily determine the components of displacement and the non-zero components of force stress as well as couple stress tensor.

\section{LOADING FUNCTION HARMONIC IN TIME}

In this case, the boundary conditions (11) take the following forms

$$
\sigma_{33}=-f\left(x_{1}\right) e^{-i \omega r}, \quad \sigma_{31}=0, \mu_{32}=0 \text { on } x_{3}=0
$$

The formula for displacements $u_{1}, u_{3}$ may be found by using the relations (2.1ab) and the equations for $\phi$ and $\psi$ in (4.21)-(4.22). Therefore we have

$$
\begin{aligned}
& u_{1}=-\frac{1}{2 \pi} \int_{-\infty}^{\infty} \int_{\frac{f}{\Delta}}^{\Delta}\left[\left(i \xi-\alpha_{1}^{*} \sqrt{\zeta_{1}}\right) \Delta_{1} e^{-x_{3} \sqrt{\zeta_{1}}}+\left(i \xi-\alpha_{2} \sqrt{\zeta_{2}}\right) \Delta_{2} e^{-x_{3} \sqrt{\zeta_{2}}}\right. \\
& \left.+\left(i \xi-\alpha_{3}^{*} \sqrt{\zeta_{3}}\right) \Delta_{3} e^{-x_{3} \sqrt{\xi_{3}}}+\left(i \xi-\alpha_{4}^{*} \sqrt{\zeta_{4}}\right) \Delta_{4} e^{-x_{3} \sqrt{\xi_{4}}}\right] e^{-i\left(x_{1} \xi+(\eta)\right.} d \xi d \eta, \\
& u_{3}=-\frac{1}{2 \pi} \int_{-\infty}^{\infty} \int_{\Delta} \frac{\hat{f}}{\Delta}\left[\left(i \xi \alpha_{1}^{*}+\sqrt{\zeta_{1}}\right) \Delta_{1} e^{-x_{3} \sqrt{\zeta_{1}}}+\left(i \xi \alpha_{2}^{*}+\sqrt{\zeta_{2}}\right) \Delta_{2} e^{-x_{3} \sqrt{\zeta_{2}}}\right. \\
& \left.+\left(i \xi \alpha_{3}^{*}-\sqrt{\zeta_{3}}\right) \Delta_{3} e^{-x_{3} \sqrt{\zeta_{3}}}+\left(i \xi \alpha_{4}^{*}-\sqrt{\zeta_{4}}\right) \Delta_{4} e^{-x_{3} \sqrt{\xi_{4}}}\right] e^{-i\left(x_{1} \xi+i \eta\right)} d \xi d \eta,
\end{aligned}
$$

We also find

$$
\begin{aligned}
\mu_{32}= & -\frac{1}{2 \pi}\left\{\left(\gamma_{0}+\varepsilon_{0}\right)-i \eta\left(\gamma_{1}+\varepsilon_{1}\right)\right\} \int_{-\infty}^{\infty} \int \frac{1}{\Delta}\left[\beta_{1}^{*} \sqrt{\zeta_{1}} \Delta_{1} e^{-x_{3} \sqrt{\zeta_{1}}}\right. \\
& \left.+\beta_{2}^{*} \sqrt{\zeta_{2}} \Delta_{2} e^{-x_{3} \sqrt{\zeta_{2}}}+\beta_{3}^{*} \sqrt{\zeta_{3}} \Delta_{3} e^{-x_{3} \sqrt{\xi_{3}}}+\beta_{4}^{*} \sqrt{\zeta_{4}} \Delta_{4} e^{-x_{3} \sqrt{\zeta_{4}}}\right] e^{-i\left(x_{1} \xi+(\eta)\right.} d \xi d \eta
\end{aligned}
$$




$$
\begin{aligned}
\hat{f}(\xi, \eta) & =\frac{1}{2 \pi} \int_{-\infty}^{\infty} \int f\left(x_{1}\right) e^{-i \omega x} e^{i\left(\xi x_{1}+\eta t\right)} d x_{1} d t=\frac{1}{2 \pi} \int_{-\infty}^{\infty} f\left(x_{1}\right) e^{i \xi x_{1}} d x_{1} \int_{-\infty}^{\infty} e^{-i(\omega-\eta)} d t \\
& =\sqrt{2 \pi} \delta(\eta-\omega) f^{*}(\xi)
\end{aligned}
$$

where

$$
f^{*}(\xi)=\frac{1}{2 \pi} \int_{-\infty}^{\infty} f\left(x_{1}\right) e^{i \xi x_{1}} d x_{1}
$$

and $\delta(x)$ is the Dirac delta function defined by

$$
\int_{-\infty}^{\infty} e^{i p t} d t=\sqrt{2 \pi} \delta(p)
$$

Substitution of (5.5) in (5.2)-(5.4) and in (4.24) gives, after integration,

$$
\begin{aligned}
& u_{1}=-\frac{e^{-i \omega x}}{\sqrt{2 \pi}} \int_{-\infty}^{\infty}\left[\frac { 1 } { \Delta } \left\{\left(i \xi-\alpha_{1}^{*} \sqrt{\zeta_{1}}\right) \Delta_{1} e^{-x_{3} \sqrt{\zeta_{1}}}+\left(i \xi-\alpha_{2} \sqrt{\zeta_{2}}\right) \Delta_{2} e^{-x_{3} \sqrt{\zeta_{2}}}\right.\right. \\
& \left.\left.+\left(i \xi-\alpha_{3}^{*} \sqrt{\zeta_{3}}\right) \Delta_{3} e^{-x_{3} \sqrt{\zeta_{3}}}+\left(i \xi-\alpha_{4}^{*} \sqrt{\xi_{4}}\right) \Delta_{4} e^{-x_{3} \sqrt{\xi_{4}}}\right\}\right]_{\eta-\omega} f^{*}(\xi) e^{-i \xi x_{1}} d \xi, \\
& u_{3}=-\frac{e^{-i \omega x}}{\sqrt{2 \pi}} \int_{-\infty}^{\infty}\left[\frac { 1 } { \Delta } \left\{\left(i \xi \alpha_{1}^{*}+\sqrt{\zeta_{1}}\right) \Delta_{1} e^{-x_{3} \sqrt{\zeta_{1}}}+\left(i \xi \alpha_{2}^{*}+\sqrt{\zeta_{2}}\right) \Delta_{2} e^{-x_{3} \sqrt{\zeta_{2}}}\right.\right. \\
& \left.\left.+\left(i \xi \alpha_{3}^{*}+\sqrt{\zeta_{3}}\right) \Delta_{3} e^{-x_{3} \sqrt{\xi_{3}}}+\left(i \xi \alpha_{4}^{*}+\sqrt{\xi_{4}}\right) \Delta_{4} e^{-x_{3} \sqrt{\xi_{4}}}\right\}\right]_{\eta=\omega} f^{*}(\xi) e^{-i \xi x_{1}} d \xi, \\
& \mu_{32}=-\frac{e^{-i \omega x}}{\sqrt{2 \pi}}\left\{\left(\gamma_{0}+\varepsilon_{0}\right)-i \eta\left(\gamma_{1}+\varepsilon_{1}\right)\right\} \int_{-\infty}^{\infty}\left[\frac { 1 } { \Delta } \left\{\beta_{1}^{*} \sqrt{\zeta_{1}} \Delta_{1} e^{-x_{3} \sqrt{\zeta_{1}}}\right.\right. \\
& \left.\left.+\beta_{2}^{*} \sqrt{\zeta_{2}} \Delta_{2} e^{-x_{3} \sqrt{\zeta_{2}}}+\beta_{3}^{*} \sqrt{\zeta_{3}} \Delta_{3} e^{-x_{3} \sqrt{\zeta_{3}}}+\beta_{4}^{*} \sqrt{\zeta_{4}} \Delta_{4} e^{-x_{3} \sqrt{\zeta_{4}}}\right\}\right]_{\eta=\omega} f^{*}(\xi) e^{-i \xi x_{1}} d \xi, \\
& \theta=\frac{e^{-i \omega x}}{\sqrt{2 \pi}} \int_{-\infty}^{\infty}\left[\frac{1}{\Delta}\left\{\dot{\gamma_{1}} \Delta_{i} e^{-x_{3} \sqrt{\zeta_{1}}}+\dot{\gamma}_{2}^{*} \Delta_{2} e^{-x_{3} \sqrt{\zeta_{2}}}+\dot{\gamma}_{3} \Delta_{3} e^{-x_{3} \sqrt{\zeta_{3}}}+\dot{\gamma}_{4} \Delta_{4} e^{-x_{3} \sqrt{\xi_{4}}}\right\}\right]_{\eta-\omega} f^{*}(\xi) e^{-i \xi x_{1}} d \xi
\end{aligned}
$$

In particular, when the applied load is a horizontal concentrated force acting at the origin, that is $f\left(x_{1}\right)=P \delta\left(x_{1}\right)$ so that $f^{\prime}(\xi)=\frac{P}{\sqrt{2 \pi}}$. Hence solutions (5.8)-(5.11) assume simpler forms. The upshot of this analysis is that solutions modified by gravity, viscosity and temperature field.

\section{CLOSING REMARKS}

With regard to the general character of the harmonic loading function utilized as a disturbance in the theory, it can be added as a concluding remark that the present analysis is sufficiently general, and in addition, it incorporates other forms of harmonic disturbances of physical interest.

\section{Acknowledgement}

This research was partially supported by the University of Central Florida. 


\section{REFERENCES}

[ 1] Eringen, A.C.: Proc. 9th Mid. Mech. Conf. New York (1967) 24-40.

[ 2] Eringen, A.C.: Edited by H. Liebowitz, Acad. Press, New York (1968) 621-720.

[3] Eringen, A.C.: J. Math. Mech. 15(1966) 909-924.

[ 4] Eringen, A.C.: Int. J. Eng. Sci. 5(1967) 191-204.

[ 5] Sengupta, P.R. and Roy, S.K.: Bull. Pol. Acad. Scs. Tech. Scs, Vol 34, No. 1-2 (1986) 37-47.

[ 6] Sengupta, P.R. and Dey, S.N.: Bull. Pol. Ac. Tech. 22(1974) 213-222.

[ 7] Sengupta. P.R. and Acharya, D.P.: Int. J. Eng, Sci. 15(1967) 211-217.

[ 8] Chadha, T.K., Kumar, R. and Debnath, L.: Internat. J. Math. Math Sci. 10(1987) 187-198.

[ 9] Kumar, R., Gogna, M.L. and Debnath, L.: Internat. J. Math. \& Math Sci. 13(1990) 363-372.

[10] Myint-U, T. and Debnath, L.: Partial Differential Equations for Scientists and Engineers (Third Edition) North Holland, (1987). 


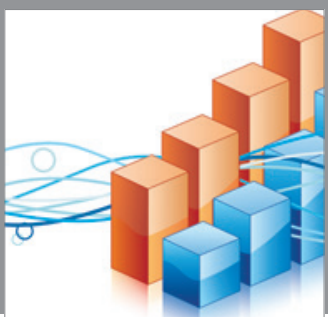

Advances in

Operations Research

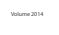

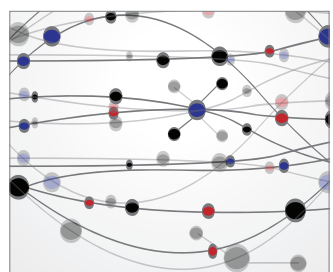

\section{The Scientific} World Journal
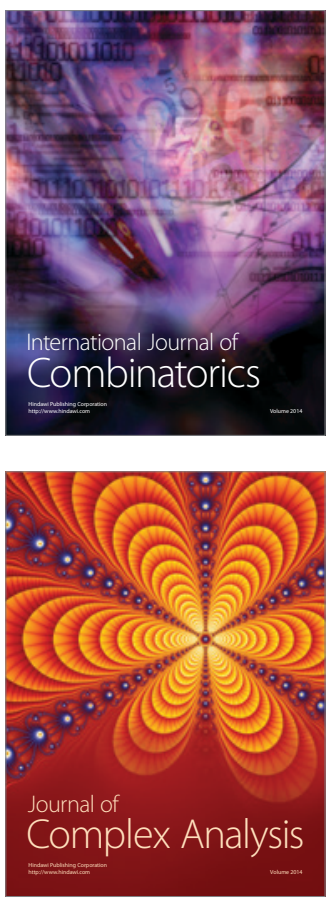

International Journal of

Mathematics and

Mathematical

Sciences
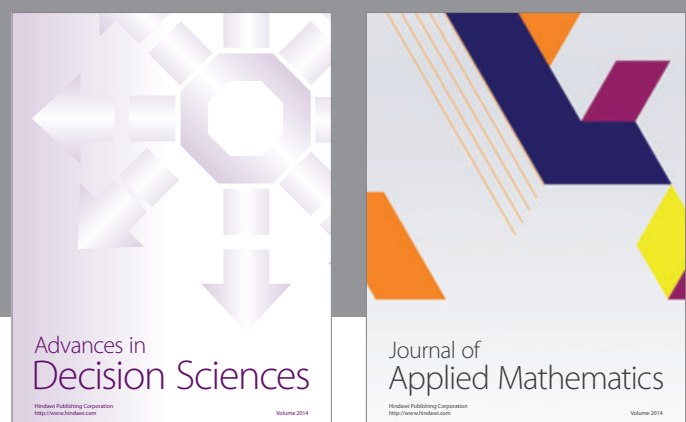

Journal of

Applied Mathematics
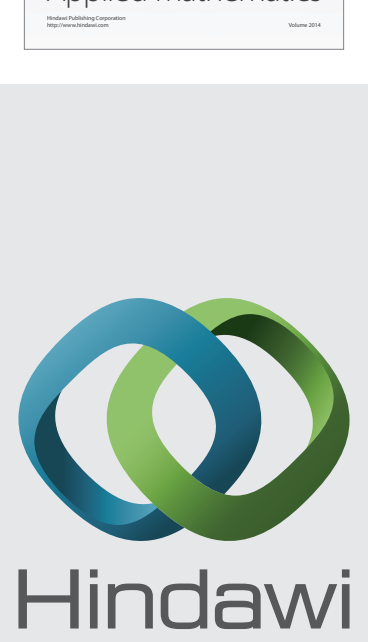

Submit your manuscripts at http://www.hindawi.com
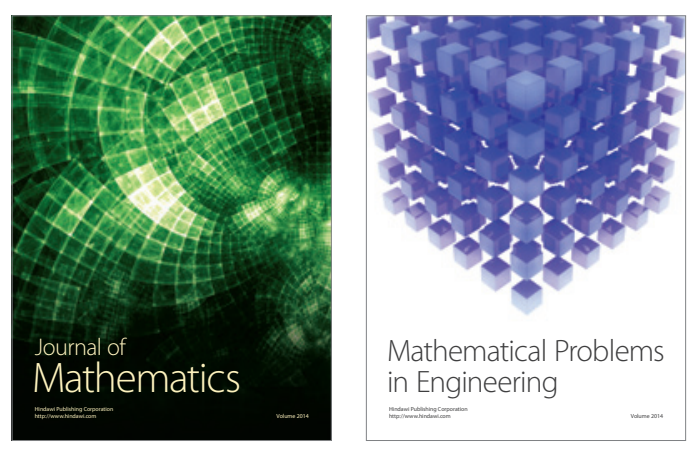

Mathematical Problems in Engineering
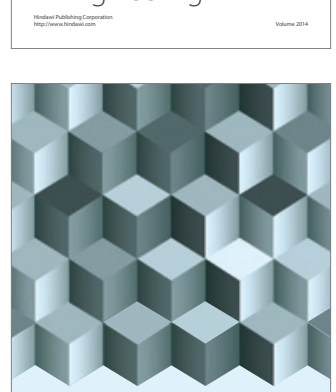

Journal of

Function Spaces
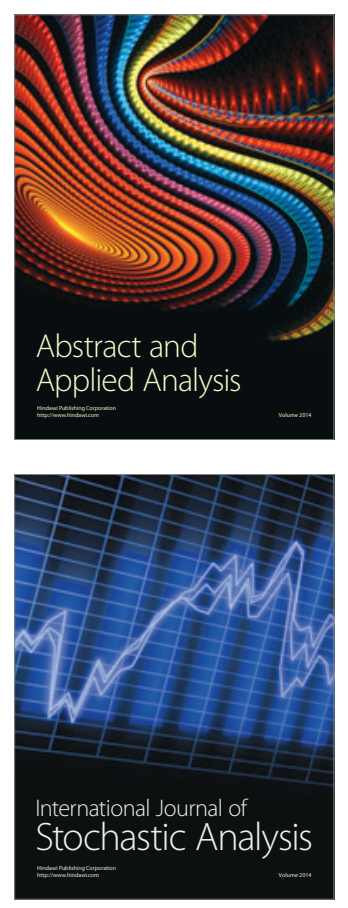

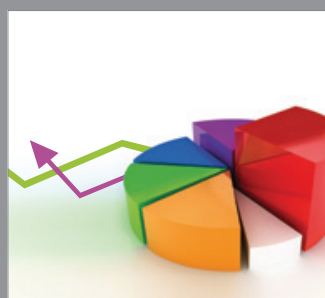

ournal of

Probability and Statistics

Promensencen
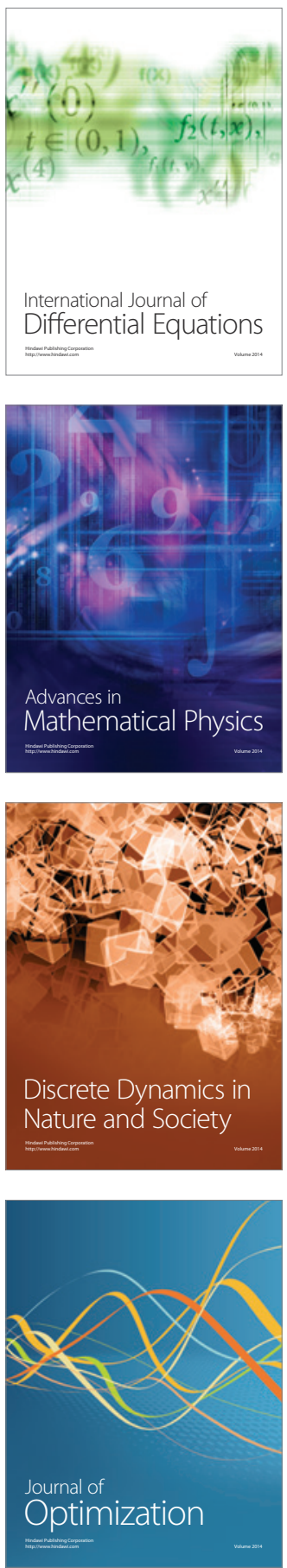